Pacific Journal of Mathematics

ON CURVATURE OPERATORS OF BOUNDED RANK 


\title{
ON CURVATURE OPERATORS OF BOUNDED RANK
}

\author{
JAAK VILMS
}

A curvature operator, that is, a linear map $R: \Lambda^{2} V \rightarrow$ $\Lambda^{2} V$, has bounded rank $2 r$ if it maps simple bivectors into bivectors of rank $\leqq 2 r$. It is shown here that this condition is equivalent to the following:

$$
\sum R\left(x_{i_{1}} \wedge y_{1}\right) \wedge \cdots \wedge R\left(x_{i_{r+1}} \wedge y_{r+1}\right)=0
$$

for all $x_{1}, \cdots, x_{r+1}, y_{1}, \cdots, y_{r+1}$ in $V$, with the sum taken over all permutations $\left(i_{1}, \cdots, i_{r+1}\right)$ of $(1,2,3, \cdots, r+1)$. An application to Riemannian geometry is given.

1. Introduction. The Riemann curvature tensor has been studied in many different algebraic contexts. In particular, it can be formulated as a linear map $R: \Lambda^{2} V \rightarrow \Lambda^{2} V$, called the curvature operator, where $V$ is a real $n$-dimensional vector space and $\Lambda^{2} V$ is its associated space of bivectors.

The concept of bivector rank is reviewed in $\S 2$. Our main result appears as Theorem 3.4 in $\S 3$. The application to Riemannian geometry is given in $\S 4$. The reader is referred to [1] and [2] for background material in exterior algebra.

The author wishes to thank Professor Marvin Marcus for supplying an elegant proof for Theorem 3.4.

2. The rank of a bivector. The bivector space $\Lambda^{2} V$ is isomorphic to the space $o(V)$ of linear maps $V \rightarrow V$ which are skew-symmetric with respect to any fixed inner product on $V$. Namely, choose a basis $e_{1}, \cdots, e_{n}$ of $V$. Then for arbitrary $\alpha \in \Lambda^{2} V$ we have $\alpha=$ $\Sigma a^{2 j} e_{i} \wedge e_{j}$, where the sum is taken either over $1 \leqq i<j \leqq n$, or over $i, j=1, \cdots, n$ with the understanding that $a^{j i}=-a^{i j}$ (and the $a^{i j}$ are divided by 2). The linear map $A: V \rightarrow V$ defined by $A e_{i}=$ $\Sigma a^{2 j} e_{j}$ is skew-symmetric with respect to any inner product for which the basis $e_{1}, \cdots, e_{n}$ is orthonormal. It is easy to check that if a different basis is chosen, the range of $A$ still stays the same; hence, $U_{\alpha}=A(V)$ is a uniquely defined subspace of $V$ associated to $\alpha$. The rank of $\alpha$ is simply the rank of such a corresponding linear $\operatorname{map} A \in o(V)$, i.e., $\operatorname{rank}(\alpha)=\operatorname{dim} U_{\alpha}$.

Note $\operatorname{rank}(\alpha)=0$ means $\alpha=0$. Bivectors of minimal nonzero rank, that is, of rank 2 , are called simple or decomposable.

We shall need some equivalent definitions of the rank of $\alpha$, expressed in the context of $\Lambda^{2} V$ rather than $o(V)$. These facts are summarized as follows. 
Proposition 2.1. Let $\alpha \in \Lambda^{2} V, \alpha \neq 0$.

(a) Rank $(\alpha)=2 r$ if and only if there exist independent vectors $x_{1}, \cdots, x_{2 r}$ such that

$$
\alpha=x_{1} \wedge x_{2}+\cdots+x_{2 r-1} \wedge x_{2 r} .
$$

(b) Rank $(\alpha)=2 r$ if and only if $\alpha^{r} \neq 0$ and $\alpha^{r+1}=0$.

(c) The rank of $\alpha$ is the smallest dimension of any subspace $U \subset V$ such that $\Lambda^{2} U$ contains $\alpha$.

(d) The rank of $\alpha$ is twice the smallest number of terms in any expression of $\alpha$ as a sum of simple bivectors.

Proof.

(a) Write $\alpha=\Sigma a^{i j} e_{i} \wedge e_{j}$, with the sum taken over $1 \leqq i<j \leqq$ $n$. Since $\alpha \neq 0$ by hypothesis, some $a^{i j}$ must be nonzero; hence the basis vectors $e_{i}$ can be relabeled to obtain $a^{12} \neq 0$. Set

$$
x_{1}=a^{12} e_{i}-\sum_{3 \leqq j} a^{2 j} e_{j}, \quad x_{2}=e_{2}+\sum_{3 \leq \imath} \frac{a^{1 i}}{a^{12}} e_{i} .
$$

Then the expression $\alpha=\sum a^{i j} e_{i} \wedge e_{j}$ can be rewritten as

$$
\begin{aligned}
\alpha=x_{1} \wedge & x_{2}+\sum_{3 \leqq i<j} a^{i j} e_{i} \wedge e_{j}-\sum_{3 \leqq i, j} \frac{a^{1 i} a^{2 j}}{a^{12}} e_{i} \wedge e_{j} \\
& =x_{1} \wedge x_{2}+\sum_{3 \leqq i<j} \frac{1}{a^{12}}\left(a^{12} a^{i j}-a^{1 i} a^{2 j}+a^{1 j} a^{2 j}\right) e_{i} \wedge e_{j} \\
& =x_{1} \wedge x_{2}+\sum_{3 \leqq i<j} \frac{1}{a^{12}}(\alpha \wedge \alpha)^{12 i j} e_{i} \wedge e_{j} \\
& =x_{1} \wedge x_{2}+\alpha_{1} .
\end{aligned}
$$

Note that $x_{1}, x_{2}, e_{3}, \cdots, e_{n}$ are linearly independent and that $\alpha_{1} \in$ $\Lambda^{2}\left\{e_{3}, \cdots, e_{n}\right\}$ (brackets $\{\cdots\}$ denote span).

Now an induction can be performed. If $\alpha_{1}=0$, we are done. If $\alpha_{1} \neq 0$, relabel the $e_{i}$ for $3 \leqq i$ to make $a_{1}^{34} \neq 0$. The above procedure is then repreated on $\alpha_{1}$ to get

$$
\begin{aligned}
\alpha_{1} & =x_{3} \wedge x_{4}+\sum_{5 \leqq i<j} \frac{1}{a^{34}}\left(\alpha_{1} \wedge \alpha_{1}\right)^{34 i j} e_{i} \wedge e_{j} \\
& =x_{3} \wedge x_{4}+\alpha_{2} .
\end{aligned}
$$

Thus $\alpha=x_{1} \wedge x_{2}+x_{3} \wedge x_{4}+\alpha_{2}$, with $x_{1}, \cdots, x_{4}, e_{5}, \cdots, e_{n}$ linearly independent, and $\alpha_{2} \in \Lambda^{2}\left\{e_{5}, \cdots, e_{n}\right\}$. Eventually, one of the $\alpha_{k}$ 's is zero, since we run out of $e_{i}^{\prime}$ 's to operate on. Hence $\alpha=x_{1} \wedge$ $x_{2}+\cdots+x_{2 r-1} \wedge x_{2 r}$, for some $2 r$. Since the vectors $x_{1}, \cdots, x_{2 r}$ are independent, $2 r \leqq n$.

Note that $\alpha \in \Lambda^{2}\left\{x_{1}, \cdots, x_{2 r}\right\}$. Moreover, if we extend $x_{1}, \cdots, x_{2 r}$ 
to a basis of $V$, then in this basis the coordinates of $\alpha$ are given by $a^{12}=a^{34}=a^{56}=\cdots=1, a^{21}=a^{43}=a^{65}=\cdots=-1$, all other $a^{i j}=$ 0 . Hence for this basis the vectors $A e_{i}$ are given by $A e_{2 k-1}=x_{2 k}$, $A e_{2 k}=-x_{2 k-1}$. It follows that $U_{\alpha}=\left\{x_{2},-x_{1}, x_{4},-x_{3}, \cdots, x_{2 r},-x_{2 r-1}\right\}=$ $\left\{x_{1}, \cdots, x_{2 r}\right\}$, and therefore $\operatorname{rank}(\alpha)=2 r$. This proves (a).

(b) The power $\alpha^{r}$ stands for the exterior product $\alpha \wedge \cdots \wedge \alpha$ where $\alpha$ occurs $r$ times. Let us substitute the "canonical" expansion given in part (a), $\alpha=x_{1} \wedge x_{2}+\cdots+x_{2 r-1} \wedge x_{2 r}$, into this product; notice that it has exactly $r$ summands. Since $x \wedge x=0$, the nonzero terms of the product $\alpha^{r}$ are obtained by choosing a different summand $x_{2 i-1} \wedge x_{2 i}$ from each $\alpha$ and multiplying these together. Since the exterior product of bivectors is commutative, each of these terms equals $x_{1} \wedge x_{2} \wedge \cdots \wedge x_{2 r-1} \wedge x_{2 r}$. Now there are $r$ ! of these terms, since a typical term can be built up in $r$ ! different ways. Therefore $\alpha^{r}=r !\left(x_{1} \wedge \cdots \wedge x_{2 r}\right)$. Since the $x_{i}$ are independent, we see that $\alpha^{r} \neq 0$; and also $\alpha^{r+1}=0$, since each term of the product $\alpha^{r} \wedge \alpha$ contains a factor of form $x \wedge x$.

On the other hand, suppose $\alpha^{s} \neq 0, \alpha^{s+1}=0$, and let rank $\alpha=2 r$. Then the above argument gives $\alpha^{r} \neq 0, \alpha^{r+1}=0$. If $s<r$, then $s+1 \leqq r$, so $\alpha^{s+1}=0$ contradicts $\alpha^{r} \neq 0$; and if $r<s$, then $r+1 \leqq s$, so $\alpha^{r+1}=0$ contradicts $\alpha^{s}=0$. Therefore only $s=r$ is possible. This proves (b).

(c) Let $s$ be the smallest dimension of any subspace $U \subset V$ such that $\alpha \in \Lambda^{2} U$. Let $e_{1}, \cdots, e_{k}$ be a basis of $U_{\alpha}$ such that $e_{1}, \cdots$, $e_{n}$ is a basis of $V$. Hence each $A e_{i}=\sum a^{i j} e_{j}$ is a linear combination of $e_{1}, \cdots, e_{k}$ only, so that no nonzero term with $e_{j}, j>k$, appears in these sums. Since $a^{j i}=-a^{i j}$, this means that the coefficients $a^{i j}$ which involve $i, j>k$ must all vanish. Therefore the expression $\alpha=\sum a^{i j} e_{i} \wedge e_{j}$ reduces to a sum over $i, j=1, \cdots, k$, whence $\alpha \in$ $\Lambda^{2} U_{\alpha}$. This implies $s \leqq k$.

Conversely, by definition of $s$, there is a basis $b_{1}, \cdots, b_{2}, b_{s+1}, \cdots$, $b_{n}$ of $V$ such that $\alpha=\sum x^{i j} b_{i} \wedge b_{j}$, summed over $1 \leqq i<j \leqq s$. Taking this as a sum over all $i, j=1, \cdots, n$, we see that $x^{i j}=0$ for $i, j>s$. Hence for this basis we have $A e_{i}=\sum x^{i j} b_{j}$, summed over $1 \leqq j \leqq s$, which implies that $U_{\alpha} \subset\left\{b_{1}, \cdots, b_{s}\right\}$. Therefore $k \leqq s$, and thus $k=s$. This proves (c).

(d) By (a), a simple bivector is of form $x_{1} \wedge x_{2}$. The required statement follows directly from (a) and (c).

CoROLLARY 2.2 .

(a) A bivector $\alpha$ is simple if and only if $\alpha \wedge \alpha=0$.

(b) If $\alpha=\sum a^{i j} y_{i} \wedge y_{j}, i, j \leqq p$, then rank $(\alpha) \leqq p$; and if the $y_{i}$ are linearly dependent, then $\operatorname{rank}(\alpha)<0$.

(c) If $\alpha=y_{1} \wedge y_{2}+\cdots+y_{2 r-1} \wedge y_{2 r}$, then $\operatorname{rank}(\alpha)$ is $=2 r$ or 
is $<2 r$ as the $y_{i}$ are linearly independent or dependent.

Proof. Part (a) is clear. For (b), note $\alpha \in \Lambda^{2} U_{y}$ where $U_{y}=$ $\left\{y_{1}, \cdots, y_{p}\right\}$. Now $\operatorname{rank}(\alpha) \leqq \operatorname{dim} U_{y}$ by Proposition 2.1 (c). If the $y_{i}$ are dependent, $\operatorname{dim} U_{y}<p$. Hence $\operatorname{rank}(\alpha)<p$. For (c) note that if $y_{i}$ are dependent, then $\operatorname{rank}(\alpha)<2 r$ by (b). On the other hand, if the $y_{i}$ are independent, then $\operatorname{rank}(\alpha)=2 r$ by Proposition 2.1 (a).

3. Curvature operators of bounded rank. The space $\Lambda^{2} V$ is a disjoint union of the subsets of bivectors of the different possible ranks $2,4, \cdots, 2[n / 2]$. We wish to consider how a curvature operator $R: \Lambda^{2} V \rightarrow \Lambda^{2} V$ maps the simple bivectors.

The image of a simple bivector is a bivector having a certain rank. At worst, this rank is $2[n / 2]=n-1$ or $n$ (as $n$ is odd or even), but it could be a smaller number. Let us say that a curvature operator $R$ has bounded rank $2 r$ if the image of each simple bivector has rank $\leqq 2 r$. This means that the range $R\left(\Lambda^{2} V\right)$ is contained in the union of the sets of vectors of ranks $2,4, \cdots, 2 r$. Our purpose here is to give a characterization for curvature operators $R$ of bounded rank $2 r$.

Curvature operators of bounded rank 2 are those that map simple bivectors into simple bivectors, or in other words, preserve decomposability; they were studied in [3] and [4]. We first state some results concerning this special case.

Proposition 3.1. If a curvature operator $R$ has bounded rank 2 , then it maps bivectors of rank $2 r$ into bivectors of rank $\leqq 2 r$, for all $r$.

Proof. Consider a bivector $\alpha$ of rank $2 r$. By Proposition 2.1 (a) it can be written as $\alpha=x_{1} \wedge x_{2}+\cdots+x_{2 r-1} \wedge x_{2 r}$. Since $R$ is linear, $R \alpha=R\left(x_{1} \wedge x_{2}\right)+\cdots+R\left(x_{2 r-1} \wedge x_{2 r}\right)$. But each of these terms is a simple bivector; hence $R \alpha=y_{1} \wedge y_{2}+\cdots+y_{2 r-1} \wedge y_{2 r}$ for suitable $y_{1}, \cdots, y_{2 r} \in V$. Now Corollary 2.2 implies that rank $(R \alpha) \leqq 2 r$.

TheOREm 3.2. [4, Prop. 3.1]. A curvature operator $R$ has bounded rank 2 if and only if $R\left(x_{1} \wedge x_{2}\right) \wedge R\left(x_{3} \wedge x_{4}\right)+R\left(x_{1} \wedge x_{3}\right) \wedge$ $R\left(x_{2} \wedge x_{4}\right)=0$ for all $x_{1}, x_{2}, x_{3}, x_{4} \in V$.

TheOREM 3.3. [3, Thm. 1]. Let $V$ have an inner product, suppose the curvature operator $R$ is symmetric in the induced inner product on $\Lambda^{2} V$ and is nonsingular, and let $n \geqq 5$. Then $R$ has 
bounded rank 2 if and only if $R= \pm \Lambda^{2} L$ for some linear map $L$ : $V \rightarrow V$.

Now we return to the general case and state our main theorem, which is a generalization of Theorem 3.2. Let $S_{r}$ denote the symmetric group on $r$ objects.

THEOREM 3.4. A curvature operator $R$ has bounded rank $2 r$ if and only if

$$
\sum_{\sigma \in S_{r+1}} R\left(x_{\sigma(1)} \wedge y_{1}\right) \wedge \cdots \wedge R\left(x_{\sigma(r+1)} \wedge y_{r+1}\right)=0,
$$

for all $x_{1}, \cdots, x_{r+1}, y_{1}, \cdots, y_{r+1} \in V$.

Proof. [Marvin Marcus]. By definition, $R$ has bounded rank $2 r$ if and only if $R(x \wedge y)$ has rank $\leqq 2 r$ for every $x, y \in V$. By Proposition 2.1 (b), this occurs if and only if $(R(x \wedge y))^{r+1}=0$ for all $x, y \in V$. But this in turn occurs if and only if

$$
\left[R\left(\sum_{1}^{r+1} \lambda_{i} x_{i}\right) \wedge\left(\sum_{1}^{r+1} \mu_{j} y_{j}\right)\right]^{r+1}=0
$$

for all $x_{1}, \cdots, x_{r+1}, y_{1}, \cdots, y_{r+1} \in V$ and all real $\lambda_{1}, \cdots, \lambda_{r+1}, \mu_{1}, \cdots, \mu_{r+1}$.

The left side of (1) can be considered as an $\Lambda^{2(r+1)} V$-valued polynomial in the indeterminates $\lambda_{1}, \cdots, \lambda_{r+1}, \mu_{1}, \cdots, \mu_{r+1}$. Upon expanding and collecting terms, we find that the coefficient of $\lambda_{1} \cdots \lambda_{r+1} \mu_{1} \cdots \lambda_{r+1}$ is precisely the left side of equation (1). But if a polynomial is identically zero, then all its coefficients must vanish. Therefore $R(x \wedge y)^{r+1}=0$ for all $x, y \in V$ implies (1).

On the other hand, if (1) holds for all $x_{1}, \cdots, x_{r+1}, y_{1}, \cdots, y_{r+1}$, then we can put $x_{1}=\cdots=x_{r+1}=x$ and $y_{1}=\cdots=y_{r+1}=y$, to get $R(x \wedge y)^{r+1}=0$ for all $x, y \in V$.

Theorem 3.4 can be restated in terms of a basis $e_{1}, \cdots, e_{n}$ of $V$. Let $R\left(e_{i} \wedge e_{j}\right)=R_{i j}$. Then

$$
R(x \wedge y)=\sum_{i, j} x^{i} y^{j} R\left(e_{i} \wedge e_{j}\right)=\sum_{i, j} x^{i} \tilde{y}^{j} R_{i j},
$$

since both $R$ and the exterior product are linear in their arguments. Note that the $R_{i j}$ are the columns of the matrix of $R$ in terms of basis $e_{i} \wedge e_{j}, i<j$, of $\Lambda^{2} V$.

THEOREM 3.5. A curvature operator $R$ has bounded rank $2 r$ if and only if

$$
\sum_{\sigma \in S_{r+1}} R_{i_{\sigma(1} j_{1}} \wedge \cdots \wedge R_{i_{\sigma(r+1)} j_{r+1}}=0
$$


for all $1 \leqq i_{\nu}, j_{\nu} \leqq n$.

Proof.

$$
\begin{aligned}
& \sum_{\sigma \in S_{r+1}} R\left(x_{\sigma(1)} \wedge y_{1}\right) \wedge \cdots \wedge R\left(x_{\sigma(r+1)} \wedge y_{r+1}\right) \\
& =\sum\left(R_{i_{\sigma(1)} j_{1}} \wedge \cdots \wedge R_{i_{\sigma(r+1)} j_{r+1}}\right) x_{\sigma(1)}^{i_{\sigma(1)}} \cdots x_{\sigma(r+1)}^{i_{\sigma(r+1)}} y_{1}^{j_{1}} \cdots y_{r+1}^{j_{r+1}}
\end{aligned}
$$

Now $x_{\sigma(r+1)}^{i_{\sigma(r+1)}} \cdots x_{\sigma(1)}^{i_{\sigma(1)}}=x_{1}^{i_{1}} \cdots x_{r+1}^{i_{r+1}}$. Hence this sum can be rewritten as

$$
\sum_{\substack{i_{1}, \ldots, i_{r} \\ j_{1}, \ldots, j_{r}}}\left(R_{i_{\sigma(1) j_{1}}} \wedge \cdots \wedge R_{i_{\sigma(r+1)} j_{r+1}}\right) x_{1}^{i_{1}} \cdots x_{r+1}^{i_{r+1}} y_{1}^{j_{1}} \cdots y_{r+1}^{j_{r+1}} .
$$

Now this sum is zero for all $x_{\nu}^{i_{\nu}}, y_{\nu}^{j_{\nu}}$ if and only if the coefficients $\sum_{\sigma} R_{i_{\sigma(1)} j_{1}} \wedge \cdots \wedge R_{i_{\sigma(r+1)} j_{r+1}}$ are identically zero.

CoROLlary 3.6. A curvature operator $R$ has an image bivector of rank $>2 r$ if and only if there exist integers $1 \leqq i_{1}, \cdots, i_{r+1}$, $j_{1}, \cdots, j_{r+1} \leqq n$ such that

$$
\sum_{\sigma S_{r+1}} R_{i_{\sigma(1)} j_{1}} \wedge \cdots \wedge R_{i_{\sigma(r+1)} j_{r+1}} \neq 0
$$

4. An application. Let $M^{n}$ be an $n$-dimensional Riemannian manifold and let $V$ denote the tangent space at any point $p$ of $M^{n}$. If $M^{n}$ admits local isometric embedding of a neighborhood of $p$ into Euclidean space $E^{n+r}$, then the curvature operator $R$ at $p$ decomposes into a sum $R=\Lambda^{2} L_{1}+\cdots+\Lambda^{2} L_{r}$, where the maps $L_{i}: V \rightarrow V$ are the second fundamental form operators. Hence $R(x \wedge y)=$ $L_{1}(x) \wedge L_{1}(y)+\cdots+L_{r}(x) \wedge L_{r}(y)$ for each $x, y \in V$, which implies that each $R(x \wedge y$ ) has rank $\leqq 2 r$ (by Proposition 2.1 (d)). Hence we get the following results, which are relevant for $r \leqq[n / 2]$.

LEMMA 4.1. If the neighborhood of a point in a Riemannian manifold $M^{n}$ admits isometric embedding into $E^{n+r}$, then the curvature operator at that point has bounded rank $2 r$.

THEOREM 4.2. Let $M^{n}$ be a Riemannian manifold, and set $R_{i j}=1 / 2 \sum_{k, l} R_{i j}^{k l} e_{k} \wedge e_{l}$, where $R_{i j}^{k l}$ is the curvature tensor and $e_{1}, \cdots, e_{n}$ is a basis of the tangent space at a point of $M^{n}$. If there exists a point in $M^{n}$ where

$$
\sum_{\sigma \in S_{r+1}} R_{i_{\sigma(1)} j_{1}} \wedge \cdots \wedge R_{i_{\sigma(r+1) j_{r+1}}} \neq 0
$$

for some integers $1 \leqq i_{1}, \cdots, i_{r+1}, j_{1}, \cdots, j_{r+1} \leqq n$, then $M^{n}$ cannot be isometrically immersed in $E^{n+r}$. 


\section{REFERENCES}

1. M. Marcus, Finite-dimensional Multilinear Algebra, Vols. I and II, Marcel Dekker, New York, 1974 and 1975.

2. W. Slebodzinski, Exterior forms and their application, Warszawa, Polish Sci. Pub., 1970.

3. J. Vilms, Local isometric imbedding of Riemannian n-manifolds into Euclidean $(n+1)$-space, J. Differential Geometry, 12 (1977), 197-202.

4. - Factorization of curvature operators, Trans. Amer. Math. Soc., to appear.

Received November 17, 1978 and in revised form July 10, 1979.

Colorado State UNIVERSITY

ForT Collins, CO 80523 

.././. ./FrontMatter/paper .pdf 


\section{Pacific Journal of Mathematics \\ Vol. 87, No. $2 \quad$ February, 1980}

Theagenis Abatzoglou, Unique best approximation from a $C^{2}$-manifold in Hilbert space ................................. 233

Gerald Arthur Anderson, $\Lambda$-homology cobordism bundles............. 245

Eric Bedford, Holomorphic mapping of products of annuli in $\mathbf{C}^{n} \ldots \ldots \ldots 271$

Gunnar Carlsson, On the stable splitting of $b o \wedge b o$ and torsion operations in connective $K$-theory .......................... 283

Lester Eli Dubins and David Samuel McIntyre Margolies, Naturally integrable functions ................................. 299

Leo Egghe, The Radon-Nikodým property, $\sigma$-dentability and martingales in locally convex spaces ............................. 313

Irving Leonard Glicksberg, Maps preserving translates of a function ..... 323

Hugh M. Hilden and Robert D. Little, Cobordism of branched covering

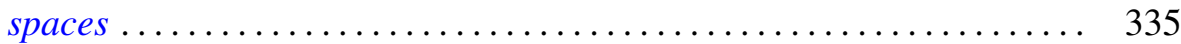

Russell Allan Johnson, Almost-periodic functions with unbounded integral .......................................... 347

Bruce Stephen Lund, The endomorphisms of a Dirichlet algebra ........ 363

John Henry McCleary, Mod $p$ decompositions of H-spaces; another approach........................................ 373

Arlan Bruce Ramsay, Subobjects of virtual groups ................ 389

Thomas R. Savage, Generalized inverses in regular rings ............ 455 Jaak Vilms, On curvature operators of bounded rank ......... 